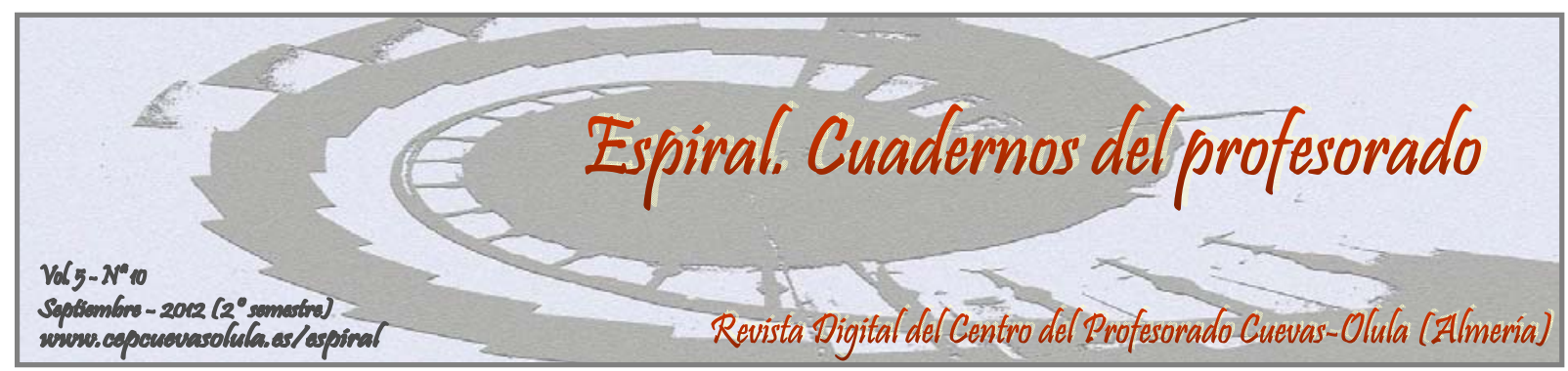

\title{
LA EVALUACIÓN DEL APRENDIZAJE EN PRIMARIA Y SECUNDARIA: LOS INDICADORES DE EVALUACIÓN
}

\section{ASSESSMENT OF LEARNING IN PRIMARY AND SECONDARY EDUCATION: EVALUATION INDICATORS}

\section{Esteban Vázquez Cano}

\author{
Dpto. Didáctica, Organización Escolar y Did. Especiales, Fac. de Educación (UNED)
}

RESUMEN: El estudio presenta una investigación sobre los procesos y procedimientos de evaluación de contenidos y competencias básicas en las etapas de primaria y secundaria en una muestra de centros educativos de Castilla-La Mancha (España). La investigación tiene como objetivos prioritarios: analizar los procesos y formas de evaluar contenidos y competencias, verificar los instrumentos de evaluación preponderantes, destacar la evaluación como predictor fundamental de calidad educativa y elemento crucial para reducir el fracaso escolar y proponer una metodología evaluadora que garantice procesos de evaluación objetivos con base en el desarrollo de indicadores de evaluación. Se ha desarrollado una metodología de corte cuantitativo y cualitativo que nos ha permitido analizar los instrumentos y la forma de afrontar la evaluación e inferir diversas conclusiones: la evaluación es uno de los aspectos del proceso de enseñanza-aprendizaje que menos ha evolucionado en los últimos años, la evaluación del proceso de enseñanza-aprendizaje es poco sistemática e incompleta. La evaluación se debe sustentar en la verificación de que el alumno ha superado los criterios de evaluación y las diferentes competencias básicas que le habilitan para conseguir los objetivos de las diferentes etapas educativas y así poder garantizar su promoción escolar y su titulación académica conforme a los preceptos normativos.

Palabras clave: Didáctica general, organización Escolar, evaluación, competencias básicas, innovación educativa.

ABSTRACT: The study presents an investigation about the processes and procedures for evaluation of contents and key competences in primary and secondary education in a sample of schools in Castilla-La Mancha (Spain). The research has as main objectives: analyze the processes and forms of evaluating content and key competences, check the prevailing assessment tools, highlighting evaluation as a main predictor of educational quality and basic to reduce school failure and propose a methodology that ensures objective evaluative processes assessment based on the development of evaluation indicators. A quantitative and qualitative method allows us to analyze tools and ways to address the assessment and infer several conclusions: the evaluation is one aspect of the teaching-learning process that has evolved less in recent years, the evaluation of teachinglearning process is piecemeal and incomplete and must be built on the verification that the student has exceeded the evaluation criteria and the different key competences that enable them to achieve the objectives of the different educational stages and, therefore, to ensure their school promotion and educational qualifications in accordance with policy prescriptions.

Key words: general didactics, school organization, evaluation, key competences, educational innovation. 
Vázquez Cano, E. (2012). La evaluación del aprendizaje en primaria y secundaria: los indicadores de evaluación. Espiral. Cuadernos del Profesorado, 5(10), 30-41. Disponible en: http://www.cepcuevasolula.es/espiral.

Fecha de recepción: 03/01/2012

Fecha de aceptación: 09/04/2012
Enviar correspondencia a: evazquez@edu.uned.es

\section{1.- INTRODUCCIÓN}

La evaluación se ha convertido en un elemento clave y fundamental en el actual sistema educativo y los parámetros para su desarrollo están variando sustancialmente para dar cabida a una evaluación más objetiva; en la que se evalúe de forma integrada los conocimientos de los alumnos según el desarrollo de los criterios de evaluación y las competencias básicas y no en la mera asimilación de contenidos. El profesorado en la actualidad es reticente — por desconocimiento o por despreocupación- a la realización de una evaluación más sistemática, rica en observaciones y aplicable a la nueva filosofía evaluativa con base en el aprendizaje competencial. Los datos que se aportan corresponden a un estudio de corte cualitativo y cuantitativo en la comunidad autónoma de Castilla-La Mancha y corroboran esta necesidad de cambio en el paradigma evaluador de ambas etapas educativas. Este artículo pretende aportar claves y estrategias para el desarrollo de una evaluación de contenidos y competencias por indicadores que genere unos procesos de evaluación más consistentes, reales y aplicables a los actuales reales decretos y decretos de currículo de las diferentes comunidades autónomas

\section{2.- MARCO TEÓRICO}

El tema de la evaluación ha sido uno de los más tratados en la bibliografía pedagógica española, si bien no siempre se han dado planteamientos conceptuales uniformes. Entre otros, destacamos los siguientes: Nevo (1989, p.15) considera que la evaluación es: el proceso que provee de razones para una correcta toma de decisiones. Lafourcade (1972, p.21) considera la evaluación como una etapa del proceso educativo que tiene por fin comprobar de modo sistemático en qué medida se han logrado los resultados previstos en los objetivos que se hubieron especificado con antelación. Rodríguez (1980, p.119) afirma que la evaluación está regida por unas especificaciones determinadas previamente, es decir, con una situación inicial, pero prevista como final. Rosales (1990, p.85), establece que es un proceso sistemático de reflexión sobre la práctica y para ello, es importante que la evaluación se base más en la práctica, en lo que realmente acontece y se da en el alumno y en la escuela a diario y que dicha reflexión y todo lo que ella conlleva (recogida de datos, análisis y evaluación de los mismos) se haga procesal y sistemáticamente. Progresiva y con criterio y no de forma puntual y desorganizada. Casanova (1995, p.55) considera que es un proceso sistemático y riguroso de recogida de datos, incorporado al proceso educativo desde su comienzo, de manera que sea posible disponer de información continua y significativa para conocer la situación, formar juicios de valor con respecto a ella y tomar las decisiones adecuadas para proseguir la actividad educativa mejorándola progresivamente y Santos (1993, p.39) considera que la evaluación es un instrumento de diálogo, comprensión y mejora de la práctica educativa.

Los posicionamientos teóricos a nivel internacional se aproximan a la evaluación desde diferentes marcos teóricos. Un grupo de estudiosos orientan los estudios hacia entender la evaluación como elemento criterial que soporta todo el sistema educativo (Banta, 2007; Allen, 2009; Browell \& Swaner, 2010). Otro grupo contextualiza la importancia de la evaluación en el aspecto competencial de los resultados (Marzano, \& Kendall, 2008; Fleischman, Hopstock, Pelczar \& Shelley, 2010; Maki, 2010). Y, una última tendencia, desarrolla principios evaluativos directamente asociados al desarrollo 
de las inteligencias y de los instrumentos de evaluación (Sternberg \& Williams, 2009; Association of American Colleges and Universities, 2010; Rhodes, 2010).

Finalmente, abordaremos este estudio tomando como referencia los estudios mencionados desde el principio fundamental de que la evaluación es un conjunto de actividades programadas para recoger información sobre la que profesores y alumnos reflexionan y toman decisiones para mejorar sus estrategias de enseñanza y aprendizaje, e introducir en el proceso en curso, las correcciones necesarias. Es un proceso sistemático de recogida de datos, incorporado al sistema general de actuación educativa, que permite obtener información válida y fiable para formar juicios de valor acerca de una situación. La evaluación necesita obtener todos los datos necesarios sobre el proceso que se sigue en la enseñanza y en el aprendizaje para regular los ritmos y para determinar lo positivo del proceso y corregir lo negativo (Vázquez, Sevillano \& Méndez, 2011 pp. 208-210).

Tradicionalmente -y todavía en muchos centros educativos es así- la metodología de evaluación es la del examen, donde se pone a prueba al alumno y se comprueba si ha adquirido o no unos contenidos impartidos. Sin embargo, en el sistema educativo actual español y europeo se propone que se evalúe todo el proceso educativo de forma objetiva a través de la concreción de los criterios de evaluación por medio de indicadores observables y medibles por tareas: acciones en clase, actitud, trabajo realizado en el día a día tanto en el aula como en casa, etc. y que el examen sea un elemento más a tener en cuenta en la calificación final.

\section{La evaluación dentro del marco normativo estatal}

La evaluación en las etapas de primaria y secundaria se regula en los actuales Reales Decretos de Enseñanzas Mínimas; respectivamente 1513/2006 para primaria (Artículos 9 y 10) y para secundaria 1631/2006 (Artículos 10 y 11). En ambas etapas se adjetiva con términos como: continua y global y aparecen los criterios de evaluación de evaluación de las áreas como el referente fundamental para valorar el grado de adquisición de las competencias básicas y la superación de los objetivos; a lo que se añade que la superación de las competencias básicas es el referente fundamental para promocionar en ambas etapas.

El desarrollo de esta tipología de la evaluación en la normativa básica actual implica, en primer lugar, que cualquier práctica de evaluación debe estar ligada necesariamente a la adopción de distintos tipos de medidas que permitan a los estudiantes avanzar en su aprendizaje. Es decir, "la evaluación ha de ser punto de referencia para adoptar decisiones que afecten a la intervención educativa, a la mejora del proceso y la adopción de medidas de refuerzo educativo o de adaptación curricular”. Simultáneamente a este seguimiento, se deben trasladar las observaciones a los propios alumnos para que la evaluación cumpla su función formativa, de corrección o refuerzo, de modo inmediato. Es necesario que el alumno participe en todo este proceso a través de la autoevaluación y la coevaluación, en unas etapas en las que se pretende impulsar la autonomía del alumnado y su implicación responsable y en la que la elaboración de juicios y criterios personales sobre distintos aspectos es una intención educativa preferente.

Tabla 1. Muestra de centros analizados. Provincia de Toledo (Castilla-La Mancha)

\begin{tabular}{ccccccc}
\hline & $\begin{array}{c}\text { Centros de } \\
\text { Infantil y } \\
\text { Primaria }\end{array}$ & $\begin{array}{c}\text { Centros de } \\
\text { Secundaria }\end{array}$ & $\begin{array}{c}\text { Centros privados- } \\
\text { concertados de } \\
\text { Infantil y } \\
\text { Primaria }\end{array}$ & $\begin{array}{c}\text { Centros privados- } \\
\text { concertados de } \\
\text { Secundaria }\end{array}$ & $\begin{array}{c}\text { Centros } \\
\text { privados }\end{array}$ & Totales \\
\hline Urbanos & 20 & 25 & 10 & 10 & 5 & 70 \\
Rurales & 20 & 10 & 0 & 0 & 0 & 30 \\
Alumnado & 1534 & 1845 & 957 & 1050 & 701 & 6087 \\
\hline
\end{tabular}

\section{3.- MÉTODO}

La finalidad de este estudio de casos múltiple es valorar el grado de incorporación de los indicadores de evaluación para el desarrollo de los procesos de evaluación del alumnado atendiendo a la consecución de los criterios de evaluación y al grado de adquisición de las competencias básicas por parte de una muestra de maestros y profesores de la comunidad autónoma de Castilla-La Mancha. El 
estudio de casos se ha realizado durante el curso 2010/2011 en cien centros educativos de la provincia de Toledo con distinto contexto sociocultural y económico con las características que se exponen a continuación:

La comparación constante que nos proponen Bogdan y Biklen (1982), y que se ha aplicado entre los distintos centros educativos de la provincia de Toledo, pretende generar teoría contrastando las hipótesis extraídas en contextos diversos. La diversidad de centros y tipologías en lugar de representar una dificultad, se convierte en un enriquecimiento metodológico que genera mayor validez a las conclusiones para disponer de una explicación generalizable en múltiples contextos. Asimismo, la comparación de estos centros es productiva por presentar las siguientes características:

- Desde un punto de vista normativo contemplan un abanico completo de la tipología de centros educativos que actualmente existen en España.

- Replicación del mismo estudio, con variabilidad y equilibrio (rural vs. urbano, primaria vs. secundaria, público vs. privado-concertado vs. privado etc.).

- Por ser centros con un número de alumnos variable, lo que le confiere variabilidad de muestras.

De esta forma, contrastando estos centros y comprobando nuestras hipótesis y conclusiones obtenidas en múltiples contextos educativos, nos facilita un método para generar teorías sustantivas, con distintos niveles de profundidad referidos a la cantidad de información recogida y a la muestra de personas implicadas: alumnos, profesores, familias e inspectores de educación. Las fases en el proceso de investigación han sido las siguientes:

1.- Se aplican los instrumentos depurados en la primera fase de inmersión en todos los centros de la provincia de Toledo; previo a este momento se realiza una validación del cuestionario e instrumento de recogida de datos por parte de los servicios de Inspección de Educación de Toledo.

2.- Se recopilan mediante visita del inspector de educación los datos y se analizan los resultados en los distintos centros de la provincia, enriqueciendo la teoría del estudio de casos y contrastando sus resultados.

3.- Se contrastan resultados en las diferentes zonas educativas y se desechan los cuestionarios o resultados poco fiables.

\section{Informantes}

- El profesorado de primaria y secundaria de los centros analizados en la provincia de Toledo (en dos cursos de primaria y dos de secundaria).

- Todos los jefes/as de departamento (etapa de secundaria) y coordinadores/as de ciclo (etapa de primaria) de los centros analizados.

- Todos los orientadores y orientadoras de cada centro.

- Todos los miembros de equipos directivos (Director/; Jefe/a de Estudios y Secretario/a) de los centros analizados.

- Una muestra de alumnos/as con el siguiente perfil: diez alumnos de cada curso y etapa, dos alumnos pertenecientes al consejo escolar del centro y veinte alumnos con todas las materias aprobadas y veinte alumnos repetidores.

- Una muestra de padres y madres de alumnos con el siguiente perfil: diez familias de cada curso y etapa distintas a la de alumnos seleccionados, dos familias pertenecientes al consejo escolar del centro y dos familias con alumnos con todas las materias aprobadas y dos con alumnos repetidores diferentes a la del punto anterior.

\section{Triangulaciones realizadas}

- Triangulación de análisis de datos (familias, alumnos, profesores y cargos en centros).

- Triangulación de técnicas en la recogida de datos (cuestionario tipo Likert y preguntas abiertas).

- Triangulación temporal-longitudinal y permanente. 
- Triangulación desde distintas fuentes o diferentes informantes claves (coordinadores, directores, estudiantes y docentes).

Para realizar el análisis de estas triangulaciones hemos adoptado los principios de Erlandson et al. (1993), es decir, estudio holístico centrado en las relaciones de los sistemas o actuantes, referenciado a aspectos personales, permanencia en el contexto, expresando los sentimientos del investigador y sus compromisos éticos. Se reelaboraron los instrumentos desde el contexto y, a posteriori, para incluso en el análisis final poderse modificar para su aplicación en un futuro en otros contextos más amplios.

Tabla 2. Cuestionario

\begin{tabular}{|c|c|c|c|}
\hline ÁMBITOS DEL CUESTIONARIO & $\begin{array}{c}\text { Inspector de } \\
\text { educación }\end{array}$ & Profesores & Familias \\
\hline \multicolumn{4}{|l|}{$\begin{array}{l}\text { ÁMBITO 1: Control y supervisión del cumplimiento normativo y } \\
\text { de la documentación académica, pedagógica y administrativa en los } \\
\text { aspectos de evaluación. }\end{array}$} \\
\hline $\begin{array}{l}\text { 1.1. ¿Informa el profesorado pormenorizadamente de los objetivos, } \\
\text { contenidos y competencias básicas conseguidas o no por el } \\
\text { alumnado? }\end{array}$ & $\mathrm{X}$ & $\mathrm{X}$ & $\mathrm{X}$ \\
\hline 1.2. ¿Aplica los criterios de evaluación del decreto de currículo? & $\mathrm{X}$ & $\mathrm{X}$ & \\
\hline 1.3. ¿Son evaluadas las competencias básicas? & $\mathrm{X}$ & $\mathrm{X}$ & \\
\hline \multicolumn{4}{|l|}{$\begin{array}{l}\text { ÁMBITO 2: Supervisión de los procedimientos e instrumentos de } \\
\text { evaluación. }\end{array}$} \\
\hline 2.1. ¿Cuál es el instrumento principal de evaluación del alumnado? & $\mathrm{X}$ & $\mathrm{X}$ & $\mathrm{X}$ \\
\hline $\begin{array}{l}\text { 2.2. ¿Utiliza el profesorado diferentes instrumentos de evaluación } \\
\text { dependiendo de la tarea o actividad? Si es Sí indicar cuáles. }\end{array}$ & $X$ & $\mathrm{X}$ & \\
\hline $\begin{array}{l}\text { 2.3. ¿Asigna le profesorado porcentajes a las pruebas de evaluación } \\
\text { efectuadas? (P. ej.: } 60 \% \text { exámenes; } 30 \% \text { actividades y trabajos y } \\
10 \% \text { actitudes). }\end{array}$ & $\mathrm{X}$ & $\mathrm{X}$ & \\
\hline
\end{tabular}

\section{Técnicas e instrumentos de recogida de información y profundidad de análisis}

Las técnicas y los instrumentos pretendieron recoger la mayor cantidad y calidad de información posible sobre el proceso de evaluación tanto de competencias básicas como de aplicación de los criterios de evaluación. A continuación, se relatan las técnicas de recogida de información proyectadas en los tres niveles de profundidad y los informantes clave:

Nivel I. Entrevista mediante cuestionario y preguntas abiertas a los equipos directivos de los centros analizados.

Nivel II. Muestra de profesores de las etapas de primaria y secundaria, mediante cuestionario, visita al aula y recogida de información. Se visitaron personalmente todos los centros analizados con una estancia que oscilaba entre los tres y los seis días. En estas visitas se pasaron los siguientes instrumentos:

- Entrevistas. Entrevistas individuales semi-estructuradas.

- Cuestionarios de opinión.

- Visita al aula del inspector para la observación.

- Recogida de información al profesorado para su posterior análisis.

- Recogida de datos e información del alumnado de las clases observadas. grupo.

Nivel III. Muestra de familias y alumnado mediante cuestionario y entrevista individual y en

En estas entrevistas, se pasaron los siguientes instrumentos:

- Entrevistas. Entrevistas individuales semi-estructuradas al alumnado y familias.

- Cuestionarios de opinión al alumnado y familias.

El cuestionario utilizado gira en torno a dos ámbitos básicos de la actuación evaluadora y supervisora en educación: 
- Ámbito 1: Control y supervisión del cumplimiento normativo y de la documentación académica, pedagógica y administrativa en los aspectos de evaluación.

- Ámbito 2: Supervisión de los procedimientos e instrumentos de evaluación.

En la tabla 2 se reflejan las preguntas del cuestionario y/o entrevistas y a quienes se les ha hecho mediante una $\mathrm{X}$.

\section{4.- DISCUSIÓN Y RESULTADOS}

Los resultados obtenidos de carácter cuantitativo se han analizado mediante el paquete estadístico SPSS. Se utilizaron análisis descriptivos y tablas de contingencia que nos facilitaban frecuencias y porcentajes de las variables analizadas (presentamos resultados de tendencia central: mediana); además estos datos han sido triangulados con la finalidad de incrementar la validez de los resultados del estudio mediante la depuración de las deficiencias intrínsecas de un solo método de recogida de datos y el control del sesgo personal del supervisor. Según Flick (2004), la "triangulación” de perspectivas aumenta la atención hacia el fenómeno en estudio. El análisis de lo resultados obtenidos arroja unos resultados muy esclarecedores según los ámbitos establecidos en el cuestionario.

a) Ámbito 1: Control y supervisión del cumplimiento normativo y de la documentación académica, pedagógica y administrativa en los aspectos de evaluación.

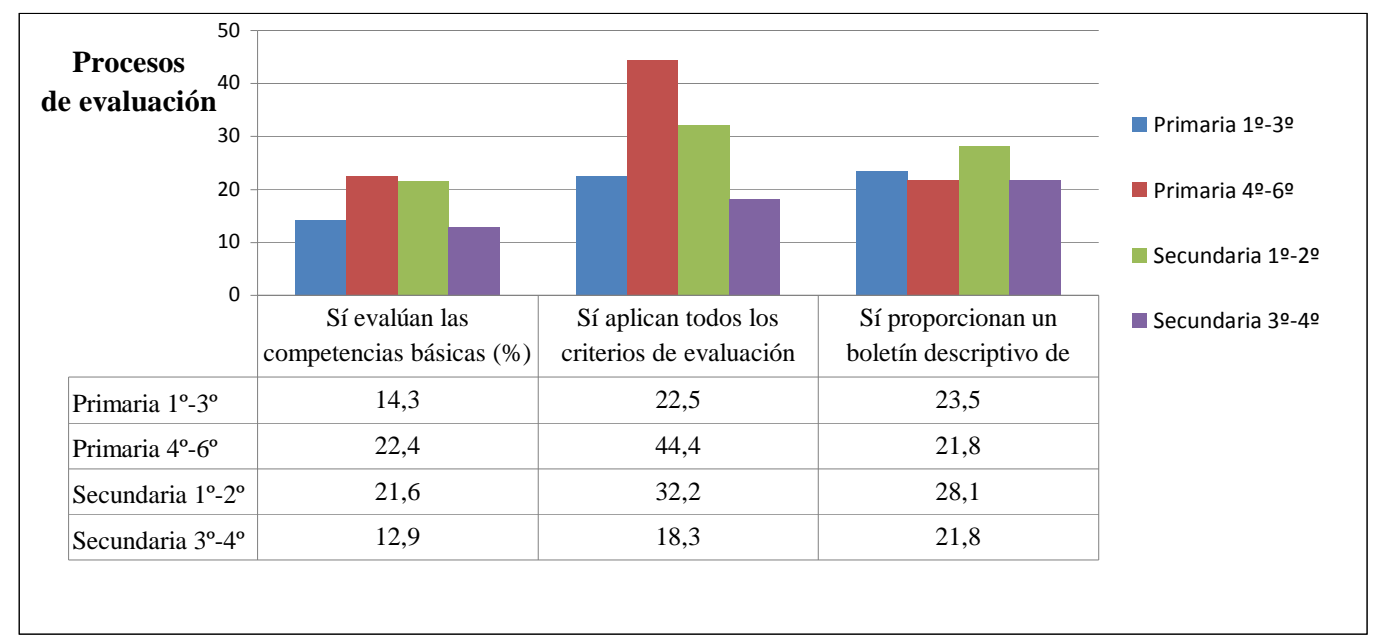

Figura 1. Control y supervisión del cumplimiento normativo y de la documentación académica, pedagógica y administrativa en los aspectos de evaluación.

En este ámbito se analizan los resultados del proceso de evaluación que realiza el profesorado atendiendo a la documentación y criterios que utiliza a la hora de evaluar. Se analizan las frecuencias asociadas a los estadísticos descriptivos (tendencia central: mediana) y se comprueba que el profesorado mayoritariamente no informa pormenorizadamente de la evaluación a las familias (88.3\%). Para ello, sólo se proporciona una nota numérica de 1 a 10 en un boletín de notas al afecto (100\%) y, en ocasiones, una anotación de las razones del mal rendimiento o comportamiento de un alumno/a (47.7\%). Asimismo, el profesorado no aplica con eficacia y en su completo desarrollo los criterios de evaluación de los correspondientes decretos de currículo (18.5\%). El profesorado no suele entregar en las diferentes evaluaciones prescriptivas durante el curso y en la evaluación final una nota desglosada del grado de consecución de las competencias básicas (92\%). Esta actuación evaluadora supone que no se desarrolla en su totalidad la prescripción normativa en materia de evaluación y que, por lo tanto, se acrecientan las diferencias entre el profesorado y el alumnado dependiendo de situaciones externas al proceso de enseñanza-aprendizaje. Esto supone que, unido a la casi inexistente evaluación del grado de adquisición y desarrollo de las competencias básicas, el sistema de evaluación queda incompleto y supone una dificultad a la hora de implantación de la nueva filosofía curricular y 
con respecto a las evaluaciones internacionales -PISA, PRILS- etc. en las que la evaluación se desarrolla desde un enfoque competencial. Asimismo, este tipo de evaluación es insuficiente, poco objetiva y poco esclarecedora para que las familias orienten y mejoren el resultado académico de sus hijos/as.

\section{b) Ámbito 2: Supervisión de los procedimientos e instrumentos de evaluación.}

\begin{tabular}{|c|c|c|c|c|}
\hline \multirow{3}{*}{$\begin{array}{cc}\text { Procesos } & 60 \\
\text { de evaluación } & 40 \\
& 20\end{array}$} & & & & \multirow{7}{*}{ 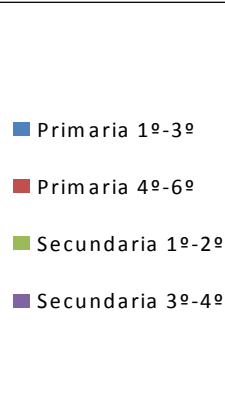 } \\
\hline & & & & \\
\hline & $\begin{array}{c}\text { Sí emplean } \\
\text { porcentajes de }\end{array}$ & $\begin{array}{l}\text { Sí emplean más de } \\
\text { tres instrumentos de }\end{array}$ & $\begin{array}{l}\text { El elemento principal } \\
\text { de evaluación es el }\end{array}$ & \\
\hline 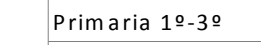 & 14,3 & 22,5 & 23,5 & \\
\hline Primaria $4 \div-60$ & 22,4 & 44,4 & 21,8 & \\
\hline Secundaria $1 \cong-20$ & 21,6 & 32,2 & 28,1 & \\
\hline Serundaria zo- 40 & 12.9 & 18.3 & 21.8 & \\
\hline
\end{tabular}

Figura 2. Supervisión de los procedimientos e instrumentos de evaluación.

En este ámbito se analizan los resultados del proceso de evaluación en relación a los procedimientos e instrumentos empleados para su desarrollo. El análisis de los datos demuestra que el principal instrumento de evaluación sigue siendo el tradicional examen de preguntas sobre contenidos teóricos de los decretos de currículo (96.5\%). Este examen sólo garantiza que, en pocos momentos puntuales, el alumnado demuestra un conocimiento sobre preguntas de contendidos teóricos pero pocas veces el examen se orienta a la demostración competencial de un contenido.

El profesorado suele orientar su didáctica a una evaluación que no se somete a variados instrumentos de evaluación dependiendo de los contenidos evaluados. De esta manera, se constata un bajo porcentaje (33.3\%) del profesorado que emplea más de tres instrumentos de evaluación. Asimismo, el profesorado mayoritariamente establece porcentajes en sus instrumentos de evaluación (92.4\%). Esto supone que se establecen prioridades no dependiendo de los contenidos y su carácter práctico o teórico sino del peso de la prueba. La actual legislación y filosofía evaluativa propugna un sistema de evaluación centrado no en el instrumento sino en la actividad o tarea que va a ser evaluada, estableciendo para ello grados de consecución (de la insuficiencia a la excelencia) lo que hace necesario que se arbitre un sistema que permita observar la consecución de los criterios de evaluación, expresados en indicadores, y el grado de adquisición y desarrollo de las competencias básicas.

\section{Aplicación de criterios de evaluación y los indicadores de desarrollo}

El actual sistema de evaluación debe ir cambiando progresivamente y con la mayor celeridad posible hacia un sistema en el que el eje de la evaluación sea la observación real y práctica de los criterios de evaluación. Estos criterios de evaluación establecen de alguna manera el tipo y grado de aprendizaje que se espera hayan alcanzado los alumnos con respecto a las capacidades implícitas en los objetivos. El modelo de evaluación a partir de criterios secuenciados en indicadores permite - tanto al alumno como al profesor - determinar los avances o retrocesos del proceso de enseñanza-aprendizaje.

Los criterios de evaluación que aparecen en los currículos oficiales deben adecuarse al contexto del centro - es decir, a la realidad concreta en la que van a aplicarse-, y secuenciarse en función de los Objetivos Generales de Etapa y de Área, así como de los contenidos mínimos que se hayan establecido para cada ciclo y curso en las correspondientes programaciones didácticas. Para ello, pueden llevarse a cabo las siguientes actuaciones:

- Matizar, en un determinado criterio de evaluación, qué capacidades se esperan desarrollar, así como en qué tipos de contenidos se concretan dichas capacidades. 
- Especificar con toda claridad el tipo y grado de aprendizaje que se pretende que el alumno alcance por medio preferentemente de tareas competenciales.

- Determinar los aprendizajes considerados mínimos y, a partir de ellos, fijar diferentes niveles, a fin de poder atender a la diversidad de ritmos de aprendizaje de los alumnos.

Los criterios de evaluación constan de dos partes: un enunciado, en el que se establece el tipo de aprendizaje y el grado en que debe ser alcanzado; y una explicación, en la que se exponen, con más detalle, los aspectos contemplados en el enunciado y, de esta manera, se evitan interpretaciones subjetivas acerca de lo que se pretende evaluar. A este desarrollo, se le denomina indicador de evaluación, que posteriormente podrá ser contextualizado, ampliado y concretado por el departamento didáctico o ciclo en el desarrollo de los procesos de evaluación. El aprendizaje al que se refiere un determinado criterio de evaluación debe graduarse de forma progresiva, para poder así determinar, en cada momento, cuál es la situación real del alumno en función de las capacidades que se pretenden desarrollar y, en su caso, poder acceder a niveles de aprendizaje superiores. En este sentido, en los criterios de evaluación se recogen, de manera inequívoca, indicadores de grado que ayudan a fijar el nivel básico de aprendizaje que se pretende que el alumno alcance. Por otra parte, y aun cuando se hayan alcanzado los contenidos básicos contemplados en un determinado criterio, la gradación de los criterios de evaluación permite contemplar los diferentes niveles de aprendizaje de los alumnos, lo que sin duda facilita la asignación de calificaciones (y, si fuera necesario, el establecimiento del nivel de apoyo aconsejable para alumnos con dificultades de aprendizaje). Desde la perspectiva del alumnado, conocer los indicadores y criterios de evaluación le sirve de guía para la elaboración de sus aprendizajes, puesto que le ayudan a comprender con claridad las tareas propuestas y, en consecuencia, las acciones que se han de ejecutar para resolverlas con éxito. En definitiva, le sirve para conocer los criterios del profesor, lo que permitirá a cada alumno autoevaluarse y regular su propio proceso de aprendizaje.

\section{Hacia una nueva evaluación del aprendizaje}

El objeto de la evaluación debe satisfacer otros objetivos más formativos que los que arroja el estudio presentado en esta investigación. Debe permitir a los que enseñan no solo la introducción de notables mejoras en el aprendizaje de los alumnos, sino también servir de instrumento crítico permanente del propio proceso educativo. Los resultados más relevantes de la investigación establecen que: no se imparten todos los contenidos de los decretos de currículo, se evalúa atendiendo a unos pocos criterios de evaluación asociados a exámenes y algún trabajo de clase o casa. El cuaderno del profesor donde se registran las participaciones del alumno es incompleto y centrado en unos pocos criterios de evaluación. Se suele reflejar la nota de los exámenes, de los trabajos y los famosos positivos o negativos por participaciones sin reflejar bien qué se está observando en relación a los criterios de evaluación.

Estos resultados deben hacer reflexionar al profesorado y orientar la práctica docente a una revisión que responda a las siguientes preguntas:

a. ¿Se imparten los contenidos de todos los bloques de contenido del currículo?

b. ¿Se aplican para la evaluación del alumnado durante un curso académico todos los criterios de evaluación? trabajos.

c. ¿ ¿ e aplican variados procedimientos de evaluación? O se suele evaluar con exámenes y

d. ¿Se evalúan de forma efectiva y continuada las competencias básicas y, para ello, se utilizan los criterios de evaluación del decreto de currículo correspondiente?

La respuesta a las anteriores preguntas debe hacer reflexionar sobre la posibilidad de impartir clase atendiendo a los contenidos de los decretos de currículo en toda su variedad y aplicar variados procedimientos de evaluación que reflejen los diferentes estilos de aprendizaje. [No sólo centrados en comprobar la memorización de determinados contenidos y enfocar la práctica educativa hacia una vertiente más práctica y funcional (competencia)]. 


\section{El desarrollo de los criterios en indicadores y la evaluación de contenidos y competencias básicas}

Podemos realizar una primera aproximación al término "indicador” a través de la definición del Diccionario de la Real Academia Española que lo define como aquello "que indica o sirve para indicar", mientras que este último término es a su vez definido como "dar a entender o significar una cosa con indicios o señales”. Un indicador no es sino una señal o indicio que permite captar y representar aspectos de una realidad que no resultan directamente asequibles al observador. De igual modo que el conductor cuenta en su tablero de instrumentos con indicadores de la temperatura del agua o del aceite o que el químico dispone en su laboratorio de indicadores que le permiten constatar la existencia de una determinada reacción que no resulta apreciable a simple vista, el científico social construye indicadores con la intención de aproximarse a diversos aspectos poco visibles de su ámbito de trabajo.

En el campo de la educación, también se han realizado varias propuestas para definir qué debe entenderse por indicador. La más clásica, además de ser una de las primeras y de las más veces repetida, es la de Oakes, autora de una obra pionera en este campo, para quien un indicador educativo es "un estadístico referido al sistema educativo, que revela algo sobre su funcionamiento o su salud" (Oakes, 1986). Su contribución consiste más bien en iluminar dicha realidad y aportar elementos de juicio para interpretarla correctamente (Bryk \& Hermanson, 1994). Es necesario reflejar la realidad de manera respetuosa y global, lo que impulsa la tendencia a ampliar el número de indicadores. La tensión no tiene siempre solución fácil, pues no debe interpretarse que exista un número mágico de indicadores necesarios para explicar adecuadamente la realidad educativa. Este aspecto ha sido interpretado y solucionado por el propio Ministerio de Educación en su reales decretos de enseñanzas mínimas y algunas comunidades autónomas que han introducido un pequeño párrafo explicativo guiando la concreción de los indicadores en el desarrollo de los procesos evaluativos, con la vinculación ya realizada a los objetivos de las diferentes etapas y al desarrollo de las competencias básicas. Como se puede ver el siguiente ejemplo:

\section{Lengua castellana y Literatura $1^{\circ}$ ESO}

1. Reconocer el propósito y la idea general en textos orales de ámbitos sociales próximos a la experiencia del alumnado y en el ámbito académico; captar la idea global de informaciones oídas en radio o en TV y seguir instrucciones poco complejas para realizar tareas de aprendizaje.

Con este criterio se trata de comprobar que los alumnos y las alumnas son capaces de parafrasear o expresar oralmente o por escrito el tema general de declaraciones públicas o informaciones de naturaleza diversa, como avisos, normas, instrucciones sencillas o noticias, así como presentaciones breves, relacionadas con temas académicos, estructuradas con claridad y con un registro divulgativo; también se pretende comprobar si se siguen instrucciones orales para realizar tareas constituidas por una secuencia de no más de tres actividades. (Real Decreto 1631/2006, de 29 de diciembre, por el que se establecen las enseñanzas mínimas correspondientes a la Educación Secundaria Obligatoria.).

Asimismo, para reducir la variabilidad en su interpretación y, si realizamos indicadores en el seno de un departamento didáctico, consideramos que los indicadores deberían cumplir con los siguientes criterios:

1.- Los indicadores usados han de estar bien definidos, indicando claramente el significado o información que se pretende obtener con su uso; es decir, ha de indicarse qué característica o variable se pretende medir.

2.- Los indicadores han de ser específicos para que nos permitan emitir un juicio de valor, en términos de mayor o menor desarrollo con respecto al criterio de evaluación que complementa.

3.- Los indicadores han de ser válidos. Para nosotros un indicador es válido cuando inequívocamente correlaciona positivamente o negativamente con la característica que queremos medir con él. Han de estar asociados a un criterio y a una o varias competencias básicas de forma inequívoca y explícita para que aporten información relevante y objetiva. 
4.- Los indicadores han de ser precisos y siempre que los empleemos nos aportarán la misma información y nos conducirán a las mismas conclusiones, con independencia del docente que utilice el indicador.

5.- Los indicadores han de tener perdurabilidad para que puedan ser mejorados en su evolución.

6.- Los indicadores han de ser comprensivos, es decir, el conjunto de todos los indicadores nos ha de proveer de información integral sobre todos los criterios de evaluación y competencias básicas.

El desarrollo de la evaluación con base en los criterios de evaluación, expresados en indicadores y en las competencias básicas requiere de un procedimiento estructurado en fases que genere una evaluación lo más objetiva y completa posible.

El primer paso requiere de una decisión consensuada dentro del departamento didáctico o coordinación de ciclo que permita decidir, entre todos sus miembros, qué criterios de evaluación son fundamentales a la hora de promocionar al alumno y que resultan, por lo tanto, referentes mínimos a la hora de evaluar. Al identificar los criterios de evaluación mínimos o fundamentales, ya llevan asociados los contenidos que vamos a trabajar durante el curso académico. Para ello, se debe valorar esta decisión conforme a su importancia y peso curricular; para posteriormente desarrollarlos en indicadores, teniendo para ello como referente el contexto socioeducativo de la zona donde se desarrolla el proceso de enseñanza-aprendizaje para lo que es clave interpretar adecuadamente el Proyecto Educativo de Centro y la Programación General Anual de la escuela en cuestión.

El segundo paso consiste en estudiar los criterios de evaluación que aparecen en los respectivos decretos de currículo de las diferentes comunidades autónomas. En muchos de ellos (como ya hemos mencionado), aparece una explicación posterior que sirve de interpretación de cada uno de los criterios de evaluación y que tendremos que adecuar a cada contexto socioeducativo. En caso de que no haya dicha explicación, tendremos que interpretar cada criterio de evaluación y adecuar su filosofía a la comunidad y contexto educativo en el que se desarrolle el proceso de enseñanzaaprendizaje. El criterio de evaluación y su explicación está referido a contenidos; por lo que tomando como referencia los criterios de evaluación, desarrollamos de forma automática los contenidos.

El tercer paso consiste en ponderar los indicadores de la insuficiencia a la excelencia; asociar tareas, actividades, trabajos y exámenes e identificar los indicadores relacionados con las competencias básicas (aunque esta labor se puede hacer de forma conjunta, el profesor es libre para desarrollar el indicador con la metodología y actividades que crea más oportuna de acuerdo con su grupo-clase). Si se elaboran indicadores que desarrollan alguna competencia, al ser evaluados, se evalúan de forma directa la consecución de competencias básicas y los contenidos correspondientes. Ejemplo de un indicador con tarea y competencia básica asociada:

Tabla 3. Relación entre Indicador, tarea y competencia.

\begin{tabular}{l}
\hline CRITERIO DE EVALUACIÓN \\
\hline 1. Reconocer el propósito y la idea general \\
en textos orales de ámbitos sociales \\
próximos a la experiencia del alumnado y en \\
el ámbito académico; captar la idea global de \\
informaciones oídas en radio o en TV y \\
seguir instrucciones poco complejas para \\
realizar tareas de aprendizaje. \\
\hline INDICADOR
\end{tabular}

1. Identifica el tema general y los hechos relevantes de un reportaje radiofónico o de TV.

COMPETENCIA
Competencia
Lingüística
Social y ciudadana

TAREA/ACTIVIDAD

En el aula de informática, audiovisuales o por medio cañón de proyección y ordenador portátil en el aula, el profesor proyecta una sección de un programa divulgativo tipo 3/14 TVE2 (10-15 minutos). Explica el concepto de idea fundamental y los recursos lingüísticos para materializarla: tipo de estructura sintáctica, léxico apropiado. El alumno realiza la práctica en el aula escrita y la expone de forma oral.

El cuarto paso consiste en arbitrar el procedimiento para asignar la nota de cada evaluación y de la evaluación final. Este es uno de los aspectos que mas controversia origina en el profesorado porque conlleva un registro más minucioso de las actividades que se realizan en el aula y fuera de ella. Es cierto que muchos profesores realizan unos dos exámenes por trimestre; cada mes y semana más o menos. Se mandan actividades y ejercicios para casa que se corrigen en clase o que el profesor entrega 
corregidos al alumnado y se registra en el cuaderno o diario de clase del profesorado, junto con un trabajo que se realiza entre el aula y casa (a veces en grupo de dos a cuatro alumnos, o de forma individual). Asimismo, se hace participar a los alumnos en clase y se refleja con positivos o con una escala numérica o similar. Esta forma de trabajo, que sigue siendo válida pero incompleta, no sistematiza los procesos de evaluación atendiendo a los criterios de evaluación. Se refleja lo que se hace de forma parcial porque no se identifica la nota de ciertos criterios de evaluación (o de muchos) y se centra en algunos contenidos que se consideran prioritarios por la forma de dar clase pero que cada profesor de un departamento o equipo didáctico suele hacer al arbitrio de una mal entendida libertad de cátedra. Para el desarrollo efectivo de estos procesos de evaluación, se precisa que el profesorado emplee unos registros de evaluación en sus cuadernos de clase más sistemáticos y organizados según los criterios de evaluación, indicadores y competencias a evaluar.

\section{5.- CONCLUSIONES}

El eje central de la educación actual derivado de la Ley Orgánica de Educación (2006) está constituido en los decretos de currículos de todas las comunidades atendiendo a: objetivos de etapa y materia, contenidos (expresados en bloques) y competencias básicas. Si nos centramos sólo en uno, desvirtuamos los demás. Si tomamos como referencia de la actuación metodológica en las clases de primaria y secundaria el criterio de evaluación, cambiaremos la metodología haciéndola más funcional (trabajaremos competencialmente). Al estar asociado cada criterio con los objetivos y directamente con los bloques de contenido del currículo; si evaluamos tomando como referencia criterios de evaluación, podremos saber si el objetivo se ha conseguido y, a la vez, si las competencias básicas implicadas se están adquiriendo por el alumnado. Esto nos hace postular unas reflexiones finales:

1. Este proceso de evaluación no es complicado pero lleva aparejado -un querer hacerlo porque es necesario- cambiar ciertas metodologías obsoletas que llevan aparejado un sistema de evaluación arcaico centrado en la repetición de contenidos principalmente teóricos del currículo de forma puntual en exámenes. En general, estudiamos inglés en el colegio e instituto desde los seis años hasta los dieciocho (hoy en día desde los 3 años) y terminamos el Bachillerato sin ser capaces de mantener con fluidez una conversación en inglés de cinco minutos. Terminamos sabiendo lo que es una oración subordinada sustantiva enunciativa no flexionada, pero no sabemos expresarnos en público con seguridad y corrección.

2. Evaluar atendiendo a criterios que hemos materializado en tareas y actividades de forma secuencial (indicadores) que nos sirven para trabajar por competencias supone un cambio metodológico profundo para dar respuesta a una sociedad cambiante en la que la memoria - aspecto esencial que hay que seguir trabajando - no es el único instrumento que nos sirve para entender e interpretar la realidad y, lo que es más importante, tenemos que tener capacidad de cambiarla porque sabemos actuar en diferentes contextos con diferentes actitudes y recursos.

3. Es cierto que para iniciar este camino se necesita la ayuda, el asesoramiento y planes de formación que den consistencia teórica y práctica a esta propuesta y en donde la administración debe jugar un papel esencial; pero igualmente resulta cierto que el docente no se puede escudar en esta falta de formación para no querer iniciar el proceso hacia una evaluación más objetiva.

4. Iniciar este cambio puede resultar una tarea tediosa y sin sentido para muchos, para otros una oportunidad de mejorar la práctica docente y reflexionar sobre la escuela que queremos y lo que esperamos de nuestros alumnos.

\section{LIMITACIONES Y PROSPECTIVA}

Esta investigación se ha desarrollado en una muestra limitada de centros de la provincia de Toledo en la Comunidad de Castilla-La Mancha. Sería deseable en futuras adaptaciones o investigaciones que se amplíe la muestra a otras comunidades autónomas para valorar el objetivo de una evaluación más objetiva y conforme a los nuevos preceptos normativas y filosofía europea. Asimismo, consideramos 
que este estudio puede ayudar tanto a futuros profesores o docentes en ejercicio a reflexionar, desarrollar e investigar sobre nuevas formas de evaluar en la enseñanza preuniversitaria.

\section{6.- REFERENCIAS BIBLIOGRÁFICAS}

Allen, M. (2009). General education assessment. San Antonio:Texas A \& M assessment Conference.

Association of American Colleges \& Universities. (2010). Engaging departments: Assessing Student Learning. Peer Review: Emerging trends and key debates in undergraduate education, 12(1).

Banta, T. (Ed.). (2007). Assessing student learning in the disciplines. San Francisco, CA: Jossey-Bass.

Bogdan, R. C. \& Biklen, S. K. (1982). Qualitative research for education: An introduction to theory and methods. Boston, MA: Allyn and Bacon, Inc.

Brownell, J. E. \& Swaner, L. E. (2010). Five high-impact practices: Research on learning outcomes, completion, and quality. Washington, D.C.: Association of American Colleges and Universities.

Bryk, A. \& Hermanson, K. (1994). Observations on the structure, interpretation and use of education indicator systems, en CERI: Making Education Count. Developing and Using International Indicators. Paris: Centre for Educational Research and Innovation-OECD, 37-53.

Casanova, A. (1995). Manual de evaluación educativa. Madrid: La Muralla.

Erlandson, D. A., Harris, E. L., Skipper, B. L. \& Allen, S. D. (1993). Doing Naturalistic Inquirí. Newbury Park, CA: Sage Publications.

Fleischman, H.L., Hopstock, P.J., Pelczar, M.P. \& Shelley, B.E. (2010). Highlights From PISA 2009: Performance of U.S. 15-Year-Old Students in Reading, Mathematics, and Science Literacy in an International Context (NCES 2011-004). National Center for Education Statistics, Institute of Education Sciences, U.S. Department of Education. Washington, DC.

Flick, U. (2004). Introducción a la investigación cualitativa. Madrid: Ediciones. Morata.

Lafourcade, P. D. (1972). Evaluación de los aprendizajes. Madrid: Cincel.

Maki, P. (2010). Assessing for learning. Sterling, VA: Stylus.

Marzano, R. J. \& Kendall, J. S. (2008). Designing \& assessing educational objectives: Applying the new taxonomy. Thousand Oaks: Corwin Press.

Nevo, D. (1989). The conceptualization of educational evaluation: An analytical review of the literature. En E. R. House (Ed.), New directions in educational evaluation. London: The Falmer Press, 15-29.

Oakes, J. (1986). Educational Indicators: a guide for policymakers. Santa Monica, CA: The Rand CorporationCenter for Policy Research in Education.

Rhodes, T. L. (2010). Assessing outcomes and improving achievement: Tips and tools for using rubrics. Washington, D.C.: Association of American Colleges and Universities.

Rodríguez, J.L. (1980). Didáctica General. I. Objetivos y evaluación. Madrid: Cincel.

Rosales, C. (1989). Perspectivas sobre evaluación educativa. Santiago de Compostela: Tórculo.

Santos, M.A. (1993). La evaluación: un proceso de diálogo, comprensión y mejora. Málaga: Aljibe.

Sternberg, R.J. \& Williams, W. M. (Eds.). (2009). Intelligence, Instruction, and Assessment. US N.J: Routledge.

Vázquez, E., Sevillano, M. ${ }^{a}$ L. \& Méndez, M. A. (2011). Programar en Primaria y Secundaria. Madrid. Pearson.

\section{Referencias normativas}

Ley Orgánica 2/2006, de 3 de mayo, de Educación.

Real Decreto 1513/2006, de 7 de diciembre, por el que se establecen las enseñanzas mínimas de la Educación primaria.

Real Decreto 1631/2006, de 29 de diciembre, por el que se establecen las enseñanzas mínimas correspondientes a la Educación Secundaria Obligatoria.

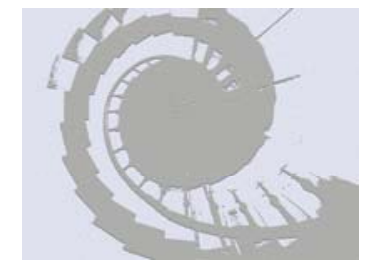

